\title{
A Collaborative Curriculum Review Process: Applicability to Higher Education Institutions
}

\author{
Andrew Alunyu Egwar \\ Busitema University \\ aalunyu@gmail.com \\ Vincent Male \\ YMCA Comprehensive Institute \\ vicelilnfif@gmail.com \\ Ismail Kamukama \\ Kyambogo University \\ kamukama.ismail@gmail.com
}

\author{
Felix Bwire \\ Busitema University \\ bwifex@gmail.com \\ Hilda Mpirirwe \\ YMCA Comprehensive Institute \\ hildakyobe@gmail.com \\ Lillian Ndagire \\ Kyambogo University \\ lillianndagire5@gmail.com
}

\author{
Irene Arinaitwe \\ Makerere University \\ iarinaitwe@cis.mak.ac.ug \\ Kenneth Baguma \\ Mbarara University \\ bagumakenneth@gmail.com \\ Assc Prof. Josephine Nabukenya \\ Makerere University \\ josephine@cit.ac.ug
}

\begin{abstract}
Curriculum review is mandatory for all higher education institutions (HEIs). The process brings together different stakeholders' expertise to evaluate and revise an existing curriculum, positioning the field of study within the current market and industry trends. Although this process is repetitive, it still remains complex, majorly due to divergent stakeholders' interests, varying levels of expertise, uncertain activity paths and multiple desired outcomes. The paper thus presents a Collaborative Curriculum Review Process (ColCuRP) to support the review of varying curricula in HEIs. We followed a mixed research approach (design science and action research) to design and evaluate the ColCuRP. It underwent four iterations during its evaluation and proved to be successful regards reduction in time for the review process, and supporting the different teams of departmental faculty to review Bachelors, Post Graduate Diploma, Masters and PhD curricula, at four HEIs in Uganda. Moreover, the ColCuRP can be used by inexperienced facilitators.
\end{abstract}

\section{Introduction}

Literally, a curriculum can be considered to be a written plan of a degree program, a syllabus, a course outline, a course study, a course guide, or a learning package [6], [14]. In fact, any HEI cannot exist without properly documented academic programs also known as a curriculum.

The curriculum review is a repetitive process (multi-step, ongoing and cyclical process) for evaluating the curriculum's effectiveness after it has been implemented [14] in an academic institution. The repetitive process goes through the cycle of convening a curriculum review committee; identifying emerging issues in specific curricula fields, weaknesses and strengths of the existing curriculum; assessing industry needs; reviewing the entire program starting from program name, program goals, objectives, expected outcomes, resources, course units, credit units, course outlines, descriptions; and updating the program [6]. Academic institutions, review their curricula to ensure each program can produce excellent students through learning experiences, generate documentation of the program quality relative to previous reviews, provide means of assessing the impact of the existing program, provide the basis for future program reviews, and meet public accountability expectations action-oriented review process [7]. Besides the curriculum review being a policy and technical issue, a process, and a product, it is always influenced by the dynamics of social changes, with a view to meeting the emerging needs and values of the society.

To accomplish the review task, the academic institutions always make use of available human resources. This is because the outsourcing option is very expensive [4], and it lacks the guarantee to produce results that positively edge the institutions' regional and international competitive stance. To achieve the noble goal of the curriculum review, the team members are faced with a challenge of identifying the existing knowledge gaps; they do this by looking at the strengths and weaknesses of the current curriculum, and establishing consensus on the courses to be modified, added or dropped in order to keep abreast with the current trends in the field of study. These tasks attract collaboration challenges such as managing the divergent interests of participants (stakeholders), their level of expertise, sequencing the activities and outputs, and agreeing on the expected outcomes [5], [8], [11], [16]. Also, achieving the main 
goal of the team work requires the services of a good facilitator [20], [21] yet most higher education institutions lack these services.

To this end, we provide a collaborative curriculum review process (ColCURP) to address the complexity challenges and time wastage faced in the review of the HIEs' academic programs. The scope of the ColCuRP is centered on the actual three key activities of the curriculum review process meeting. These include; reviewing of the relevant reference documentation/materials that guide justifying the need to review the academic program, structuring of the academic program preliminaries (program name, goals, objectives, expected outcomes, resources, grading, and admission requirements) and defining of the knowledge areas with corresponding course outlines (course units, their credit worth, and organization). In other words, the ColCuRP does not take care of the pre-review activities (such as planning for the review, obtaining the required logistics, and selecting participants) and post review activities (particularly course content detailing/description) of the curriculum review process.

The remainder of the paper is organized as follows: Section 2 discusses the current curriculum review procedure and existing challenges; in Section 3 we discuss the use of the collaboration engineering approach in designing high-value recurring/repetitive processes like the curriculum review, in HEIs. Section 4 presents the mixed research approach we followed in this study, while Section 5 presents the ColCuRP design phase in which we cover the identification of the design requirements, the actual process design (identification of the thinkLets and collaboration patterns), and the process refinement. In Section 6, the ColCuRP process testing and evaluation results are presented and discussed, and finally conclusions and future recommendations are presented in Section 7.

\section{Current Curriculum Review Procedure}

In order to meet the national regulatory body requirement for academic programs review, for example in Uganda's case, the National Council for Higher Education (NCHE); HEIs engage the services of various stakeholders during the review of their academic programs. These stakeholders include but are not limited to; academic staff, students, alumni, policy makers, academic registrar's office and quality assurance officers [11]. The HEIs go through a repetitive cycle that includes the pre-review activities, actual review activities and post-review activities after every three, four or five years, depending on the program of study. The pre-review activities include planning for the review, obtaining the required logistics, selecting and inviting the participants, and identifying and distributing relevant reference materials. The actual review meeting(s) goes through the following procedure: -

(i) Overview of the meeting agenda and goals - the Chairperson welcomes the members, provides the overview of the agenda, states the aim/goals, and the expectations of the review meeting.

(ii) Discussion of the core competencies, subject themes and emerging trends - this activity starts with the members benchmarking, identifying gaps, emerging themes and course units to add/remove/modify from the current curriculum. This session always results in prolonged debates and sometimes conflicts, that may pose a threat to the meeting cohesion as expressed in an interview by one of the respondents "if not properly resolved, comments on course units become personal issues leading to fights at departmental level".

(iii) Revision/development process - the courses are split among the faculty members to review/develop the course outlines, descriptions, aims/objectives, learning outcomes, and indicative content. They also constitute the credit units; identify reading materials, delivery methods and assessment modes. The participants then submit to the secretariat for compilation.

(iv) Secretariat of curriculum review - the secretariat compiles the reviewed curriculum for submission and onward approval processes.

Most times these review workshops drag on, consume time and sometimes provoke conflicting issues; for example, the academic participants conflicting on what courses are core or not, what courses to include or drop, and the chair dictating on the proceedings, to mention but a few.

\subsection{Challenges with the Current Curriculum Review Process}

Although the current curriculum review process is repetitive, it still remains a complex task. A task is complex if it is never fully known, not easily analyzed, goals and methods are unclear, ill-structured, ambiguous, and difficult with many parts in an intricate arrangement [5], [8]. In fact, Campbell [5] argued that "tasks having multiple paths that are imprecisely linked to several desired but conflicting outcomes are likely to be unstructured, ambiguous, and difficult", hence complex. Level of complexity is relative to abilities of the task-doer [8]. The curriculum review process is characterized as a complex task due to a number of factors among others including;

(i) The involvement of many stakeholders with divergent/conflicting interests - the curriculum review process involves a wide range of both 
internal and external stakeholders such as the academic institutions, national educational policy makers and regulators, the alumni, and industry employers [11]. Whereas problem solving requires convergence, these many actors may have divergent interests including curriculum content, program requirements, career opportunities, employable skills and financial interests, among others resulting into conflicts. The conflict of interests may increase among stakeholders who feel their inputs are superior to others; for example, Campbell [5], observed that "if achieving one desired outcome conflicts with achieving another desired outcome, complexity will increase"; meaning that the curriculum issues can be too complex to discuss with the varying stakeholders.

(ii) Participants' varying levels of expertise and interests - task complexity is relative to abilities of the task-doer [8]. Also, Nunamaker et al., [18] argue that disputes often arise from participants having incorrect or incomplete information or because of differing philosophical approaches to an issue. With varying levels of expertise, the reviewers' approaches and contributions based on incomplete or incorrect information often result into conflict among the participants.

(iii) Uncertain activity paths - in order to achieve the overall goal of a reviewed curriculum, the review activities can be approached in many different ways (paths). Unfortunately, the connection between the curriculum review activities and the desired outcomes cannot be established with certainty. Eden, et al., [8] argue that inexact or unknown means of achieving an overall goal is a characteristic of a complex task. In fact, the increase in the number of possible ways to arrive at a desired outcome increases information load, and thus it increases complexity [5].

(iv) Multiple desired outcomes - any curriculum review has multiple desired outcomes. According to Campbell [5], each of the multiple outcomes can be considered a task dimension that requires attention. The author further argues that as the number of desired outcomes of a task increases, complexity also increases [5], [8].

The main objective for collaborating on a task like the curriculum review in HEIs is to combine the expertise, insights, and mental efforts of the various stakeholders to some degree, so as to achieve a common goal [16], [20] of a reviewed curriculum. The complexities that arise must be completely eliminated or minimized in order to achieve the main goal. This can only be realized if an appropriate mechanism is put in place to harness the benefits of a facilitated meeting, even in the absence of a professional facilitator. The Collaboration Engineering approach provides such an opportunity for designing a process for repetitive and predictable patterns for collaboration among the curriculum review teams to achieve a common goal.

\section{CE Approach to Curriculum Review}

Collaboration Engineering (CE) is defined as "an approach to the design of re-usable collaboration processes and technologies meant to engender predictable and success among practitioners of recurring mission-critical collaborative tasks"[16], [21], like the curriculum review activities. Collaboration is the degree to which people combine their mental efforts so as to achieve common goals. In $\mathrm{CE}$, a collaboration process is considered as a series of activities supported by collaboration patterns and thinkLets to accomplish a goal [13].

A collaboration pattern $(\mathrm{CP})$ is a means to determining how a group moves through meeting activities (phases) to attain an agreed upon goal [13], [17]. To create a CP, "a team leader/facilitator needs to give instructions so that the team members can follow a logical sequence of actions to share and process information using certain tools" [21]. The information is codified and recorded into a reusable design pattern (thinkLet) [21]. ThinkLets are facilitation techniques (smallest unit of intellectual capital) required to create a single repeatable, predictable pattern of collaboration among people working toward a goal [4], [13]. Thus, thinkLets facilitate patterns of collaboration.

$\mathrm{CE}$ involves deploying the designs for practitioners, who are domain experts, to execute for themselves without ongoing support from professional facilitators [18], [20], [21]. In fact CE is meant to harness good facilitation techniques through the use of information and communication technology to enable collaboration between people [19]. However, skilled facilitators tend to be expensive, either through in-house training, or hired consultants [4]; thus they may only be retained for ad hoc processes. According to de Vreede and Briggs [20], CE should focus on recurring processes, rather than ad hoc processes; and building these recurring processes as a sequence of facilitation interventions, to create a pattern of collaboration [13]. Examples of successful implementation of such recurring tasks/processes are in commercial, government, and military organizations where practitioners conduct the processes for themselves without the ongoing intervention of professional facilitators [4]. We extend this to the education sector, and specifically apply it to the process of curriculum review in HEIs.

Curriculum review in an academic institution is a repetitive process of evaluating the curriculum's effectiveness after it has been implemented, updating the program so that it can produce excellent students 
through their learning experiences, establishing the basis for future program reviews, and meeting public accountability expectations for the review process [7], [14]. Thus, the curriculum review process is a missioncritical task that attempts to create substantial value, or reduces the risk of loss of substantial value of academic programs [20].

To make the best of $\mathrm{CE}$ in the curriculum review process, during the design of the process, we convert the key steps uncovered in the planning process to the five unique $\mathrm{CE}$ patterns for collaboration. These collaboration patterns have been identified by collaboration engineers to help take the group's repetitive activity to attain a common goal [13], [20]. Each of these patterns is characterized by activities that move the group from an initial phase to an end state [16], [20], [21] as explained below;

(i) Diverge: Helps groups move from having fewer concepts to having more concepts. The group generates more concepts, than previously enlisted;

(ii) Converge: Move from having many concepts to focusing on, and understanding of, a few worthy concepts. It leads to reduction of the concepts that should be given further attention;

(iii) Organize: Helps the group move from less to a better understanding of the relationships among the remaining concepts;

(iv) Evaluate: Helps the group to gain more understanding of the benefits of the concepts towards attaining a goal based on set criteria;

(v) Build Consensus: Allows the group to gain more agreement among stakeholders for mutually acceptable commitments.

Although these patterns are building blocks in designing of any repetitive process [20], they do not explicitly detail how a group could conduct a recurring collaboration process [13], [20]. Therefore, to aid inexperienced facilitators to conduct a collaborative meeting, thinkLets are required [4]. We thus used the thinkLets as a scripted collaboration activity that produces a predictable, repeatable pattern of collaboration among the curriculum review team to achieve a common review goal [13].

\section{Research Approach}

We followed a mixed research approach of both the design science (DS) and action research (AR) methods for conducting and evaluating CE efforts [15]. We followed the DS method to design the curriculum review process shown in Figure 1. In particular, we used the CE design principles [15] to explain how the DS method was followed to design an artifact (the ColCuRP) as detailed in section 5 .

Design science is an outcome based information systems research methodology, which offers specific guidelines for evaluation and interactions within research projects [15]. It embodies the creative, artistic, and goal oriented spirit within which purposeful artifacts are created [9], [10]. Though, DS has guidelines for evaluation, it is limited [9], [10], [15]. Thus, calls for a method to supplement it in order to make appropriate improvement(s) in the designed artifact. [15] argues that $\mathrm{AR}$ is a well-executed (proven) evaluation method, hence suitable for evaluating DS artifacts in CE efforts, instead of computational and mathematical evaluation methods by [9], [10].

Generally, the use of AR in CE efforts involves executing and refining the prototype collaboration process in pilots, leading to roll-out of the final process [15]. Therefore to evaluate and test the collaborative curriculum review process (ColCuRP), we followed the AR principles to evaluate a CE effort as discussed in [15]. Action research was chosen because it enables asking the 'how to' research questions, [2], [15], [22]. Our key research question was "how to improve the curriculum review process complexity in HEIs curriculum review meetings". Action research also enables testing artifacts by applying them in real-life settings [2], [15], [22]; hence the ColCuRP was tested in four HEIs environments. Action research has also been successfully used in other similar CE studies [1], [12], [16], [17].

An action research study consists of five phases; diagnosing, action planning, action taking, evaluating and specifying learning [3], [22], which [15] summarizes into four CE applicable phases, that is, planning (diagnosing and action planning), acting, observation and reflection-iterations. The planning phase involved preparations of the testing meeting sites. In the second phase (act), the actual curriculum review process sessions were conducted by the researchers and site stakeholders. During the meetings, the researchers kept on observing (using data collection instruments) whatever transpired in the meetings, with respect to the validation criteria set forward in the observation phase. Finally, the reflection phase involved analyzing the collected data (that is, what did and did not work in terms of the collaboration process) to form conclusions that we used to refine the next testing meetings and the ColCuRP. The meetings were supported by MeetingWizard Group Support System (GSS). Besides the GSS, we also used other tools, that is, the flipchart, white board markers and whiteboard.

Four academic program cases were carried out in four HEIs, and this allowed us to reflect on the process design and to improve it continuously. The participants included institutional management such as Heads of Departments, Deans and Quality Assurance officers and faculty members who were technical in a given academic subject program; while the observers and 
facilitator(s) were PhD Information Systems students who developed the ColCuRP.

During the sessions, an observation guide was used during the evaluation sessions to gather information on experiences with the ColCuRP. The cases are described below:

Case 1: The course reviewed was the Bachelor of Information Systems (BIS) at Kyambogo University (KYUS), Uganda. The collaboration meeting involved five (5) participants, six (6) observers and a facilitator and chauffeur (co-facilitator who operated the MeetingWizard GSS).

Case2: The course reviewed was the Masters in Business Administration at Team Business Institute (TBI), Uganda. The collaboration meeting involved nine (9) participants, six (6) observers and a facilitator and chauffeur.

Case 3: The course reviewed was the Post-Graduate Diploma in IT at YMCA Comprehensive Institute, Uganda. The collaboration meeting involved four (4) participants, seven (7) observers and a facilitator who was also the chauffeur.

Case4: The course reviewed was the $\mathrm{PhD}$ in Information Systems at Makerere University, Uganda. The collaboration meeting involved six (6) participants, seven (7) observers and a facilitator who was also the chauffeur.

For each case, the meetings had two major goals. The primary goal for the meetings was to test and evaluate if the ColCuRP would aid the participants to review the curriculum in the actual review phase of the curriculum review process meeting. The second goal for the meetings was to see how the collaboration technology (MeetingWizard) can supplement and quicken the collaborative curriculum review process. The purpose of the meetings from the researchers' perspective was to evaluate and refine the curriculum review process to produce a repeatable process that reduces the time for the curriculum review and can be applied by HEIs.

The purpose of the meetings from the curriculum review process perspective was to produce a generic and more certain structured path and or approach to the curriculum review process for all HEIs. The nature of participants in terms of their background knowledge and expertise had some similarities and differences. The participants in all cases had a good background in practice and lecturing course units related to the disciplines of Information Technology and Information Systems. Case 2 had two participants with Business Administration background and Case4 had five participants with $\mathrm{PhDs}$ in Information Systems. In all cases, majority of the participants had minimal background to group support technology-driven meetings.
Data was collected from four sources; direct observation, questionnaires, data logs from the MeetingWizard GSS and informal interviews.

1. Direct observation: During the group meetings, the researchers made notes of the critical incidents and questions from participants relating to the meeting process and content (for example one participant asked "can I discus with myself first, then I give my contribution?"). This helped to monitor the participants' understanding of the goals of the process, their satisfaction with the process outcomes, ability to accomplish the tasks involved in the allocated time and their perception of the GSS tool used to support the participants during the meeting.

2. Questionnaires: These were administered after each session, to get feedback on the evaluated aspects. We captured information about both the ColCuRP meeting satisfaction and the previous curricula review meetings.

3. Data logs from Meeting Wizard GSS: We stored the content from the GSS as it provided insights on the focus and clarity of the contributions made by the participants.

4. Interviews: These were held with participants at every end of the meeting to verify the responses gotten from the questionnaires.

\section{ColCuRP Designing}

This section presents the ColCuRP designing based on the requirements derived from the challenges of the existing curriculum review process. The collaboration patterns and thinkLets required to create repeatable and predictable patterns of collaboration among the curriculum review team(s) are also presented.

\subsection{The ColCuRP Process Objectives}

In order to address the complexity and to avoid the inadequacies (provocations, conflicts and delays) of the existing curriculum review process, we designed and developed a collaboration process for managing the curriculum review meeting. The ColCuRP aims at collaboratively supporting varying actors involved in the curriculum review process of varying academic programs at varying levels in HEIs to successfully facilitate their review meetings.

We designed a process with repeatable and predictable patterns for managing collaboration among the curriculum review team members. The designed ColCuRP aims to support varying curriculum review team(s) to achieve the curriculum review meeting $\operatorname{goal}(\mathrm{s})$. 


\subsection{The ColCuRP Design Requirements}

The requirements for the ColCuRP were derived from the curriculum review challenges in Section 2.1. These requirements included;

(i) Acceptable unified view of the curriculum - to overcome the challenge of divergent interests of several stakeholders, the $\mathrm{CP}$ should facilitate consensus building and agreement among participants about the wholesome characteristic of the curriculum under review.

(ii) Facilitated Group Support - with the aid of a facilitator and meeting tools, the ColCuRP should be able to allow and control communication and information sharing among participants. This overcomes the challenge of varying levels of expertise and interests among participants; hence minimizing possible conflicts and focusing the participants on the task at hand.

(iii) Structured activity paths - the $\mathrm{CP}$ should be predictable (established path between curriculum review activities and desired outcomes) and repeatable (can be used by varying sets of curriculum reviewers on varying programs in varying HEIs) with a defined set of activities so as to overcome the uncertain activity paths.

(iv) Acceptable outcomes - the ColCuRP should facilitate reaching consensus on the desirable curriculum outcomes, to solve the problem of complexity that arises from multiple desired outcomes of a given task. This guarantees a proper review of the curriculum with consideration to the reference materials and policy documents chosen to guide the review.

\subsection{The ColCuRP Collaboration Patterns and thinkLets}

During the development of the ColCuRP, the activities that were identified were converted to collaboration patterns and corresponding thinkLets to be carried out during the review sessions. A summary of the activity, collaboration pattern and thinkLet (including tools for each thinkLet) identified for an effective collaborative curriculum review meeting are presented in Table 1 of the final process design. The description that follows shows how each activity (1 to 10) was matched with the collaboration pattern and related thinkLet.

Table 1. Final Process Design for the Collaborative Curriculum Review Process (CoICuRP)

\begin{tabular}{|c|c|c|c|}
\hline Activity & $\mathbf{C P}$ & thinkLet & Tool \\
\hline $\begin{array}{l}\text { 1. Review program structure/preliminaries based on } \\
\text { NCHE minimum standards }\end{array}$ & Diverge & DirectedBrainstorm & GSS-MeetingWizard \\
\hline 2. Refine Program Structure/preliminaries & Converge & ReviewReflect & White Board \\
\hline 3. Identify core competencies & Diverge & OnePage & GSS-MeetingWizard \\
\hline 4. Build agreement on Core Competencies & Build Consensus & MoodRing & Flip Chart \\
\hline 5. Identify Subject themes/main knowledge areas & Diverge & FreeBrainstorm & GSS-MeetingWizard \\
\hline 6. Build agreement on theme list & Build Consensus & MoodRing & Flip Chart \\
\hline 7. Identify courses to add in each thematic area & Diverge \& Organize & PopcornSort & GSS-MeetingWizard \\
\hline $\begin{array}{l}\text { 8. Select courses to add, remove or modify based on } \\
\text { categories }\end{array}$ & Converge & BucketBriefing & Flip Chart \\
\hline 9. Constitute credit units (CUs) for each course unit & Evaluate & BucketWalk & Flip Chart \\
\hline $\begin{array}{l}\text { 10. Confirm preliminaries and course outlines that } \\
\text { have been reviewed/ developed }\end{array}$ & Build Consensus & MoodRing & GSS-MeetingWizard \\
\hline
\end{tabular}

Activity 1: In this activity, the participants were required to consider all the preliminary program details, leaving out only the program structure and the course descriptions. This activity translates to the diverge pattern with the DirectedBrainstorm thinkLet. This thinkLet allows the participants to be focused on one aspect of the preliminaries at a time as predefined by the facilitator and ensures that the goal of the activity is achieved.

Activity 2: This activity translates to the converge pattern, with the ReviewReflect thinkLet. This thinkLet allows the group to review and modify the contents of an existing outline, in this case the program preliminaries. The thinkLet is very helpful for reviewing the content that evolves as the process goes on. The output of this activity is a well-refined outline of all the program preliminaries.

Activity 3: This activity requires that the participants identify the core competencies required for the course. This translates to a diverge pattern; and since there are only a few contributions that can be made, the OnePage thinkLet is used. Participants work in small teams of less than 6 members to simultaneously contribute on the same page.

Activity 4: In this activity, the participants agree on the core competencies that emerge from activity 3 . The participants agree on the high-quality ideas while providing explanations as to why these ideas are better than others and need to be a core competency. The 
collaboration pattern for this activity is Build Consensus with a corresponding MoodRing thinkLet.

Activity 5: In this activity, the participants generate a list of key knowledge areas for the course, based on the core competencies. The FreeBrainstorm thinkLet is used. This thinkLet enables participants to generate a large number of ideas.

Activity 6: The participants agree on the emerging trends/knowledge areas in this activity. The MoodRing thinkLet for Build Consensus CP allows the members to agree on the list of knowledge areas, categorized by subject themes.

Activity 7: In this activity, the participants diverge by proposing the course units, and then organize them according to each theme identified in activity 6 . Both diverge and organize activities are carried out using the PopcornSort thinkLet. The thinkLet is chosen to allow the participants propose and sort course units for the themes in which they have greater expertise or interest.

Activity 8: This is a convergence activity, and it is guided by the BucketBriefing thinkLet, where the participants are asked to benchmark the courses in the current curriculum against the identified subject themes/knowledge areas, policies and other curricula. The participants list the courses to add, remove or modify based on the identified subject themes/knowledge areas and best practices in the field.

Activity 9: In this activity, participants constitute the courses \& their credit units. The activity corresponds to the Evaluate collaboration pattern with the BucketWalk as the corresponding thinkLet. This thinkLet allows the participants to ensure that each course unit has the correct credit units assigned.

Activity 10: The final activity involves building consensus among the participants on the reviewed curriculum. This Build Consensus collaboration pattern with the corresponding MoodRing thinklet enables the participants to agree on whether the goal of the entire process has been achieved and a decision is taken.

\subsection{The ColCuRP Process}

This final process as presented in Figure 1 represents the steps (meeting activities) with the corresponding collaboration patterns (CP) and appropriate thinkLets required to achieving the set objectives. The entire curriculum review process consists of three phases, that is, Pre-Curriculum Review Phase, the Curriculum Review Phase (actual curriculum review meeting) and the Post-Curriculum Review Phase.

Both the pre-review and post-review phases consist of activities that are done prior and after the review meeting. Pre-review activities include the selection of the review team(s), documents to review, and logistics among others. The post-review meeting activities cover the course descriptions and review document compilation.

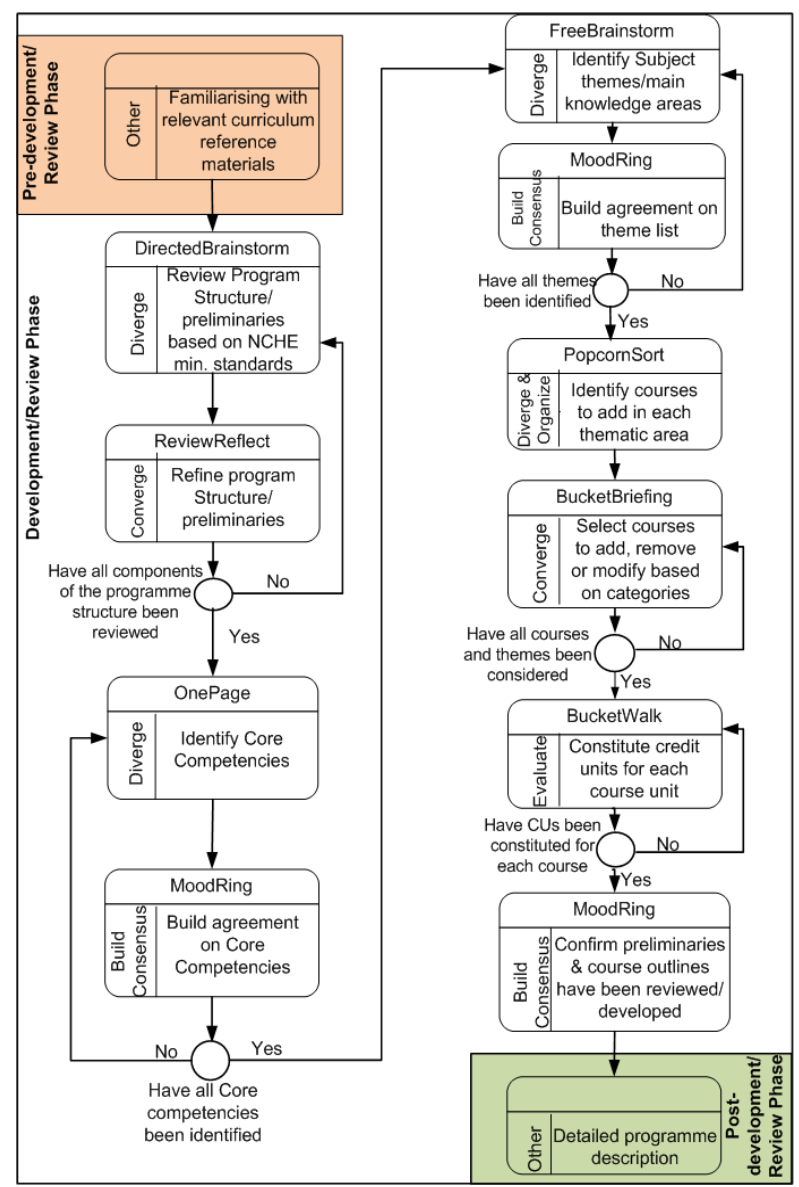

Figure 1. Collaboration Process Diagram for the Curriculum Development/Review

The actual curriculum review meeting performs three main tasks, that is, brainstorming on the needs of the academic program, structuring the academic program preliminaries and stipulating the relevant knowledge areas. Therefore, the ColCuRP process presents a set of activities, their appropriate CPs and thinkLets for achieving the objective actual curriculum review meeting. These activities include; reviewing the program structure/preliminaries; refining the program structures/preliminaries; identifying the core competencies and emerging themes; agreeing on the core competencies; identifying the subject themes/main knowledge areas; cleaning up the theme list; selecting the courses to add, remove or modify based on thematic categories; constitute the credit units for the new courses and those to be modified; and finally, confirming that the preliminaries and course outlines have been successfully reviewed/developed. 


\subsection{The ColCuRP Process Refinement}

The final process design shown in Figure 1 resulted from four design iterations that were carried out. The key modifications from the initial process design are briefly described below.

In the first process design we considered starting with the activity on "familiarize with relevant curriculum reference materials" as part of the actual curriculum review phase. However, in the discussions that followed, we agreed to have it as a pre-curriculum review activity to allow the participants enough time to prepare for the review meeting. Additionally, we had another activity on "Benchmark current curriculum against current trends, policies and other curricula", however, it was later considered to be part of the precurriculum review phase.

After the group discussions, the agreed-upon process design had "Identify and refine core competences" as the first activity, followed by "identify and refine subject themes". The activity for reviewing the program structure/preliminaries followed these activities in the initial design. However, this caused confusion during the initial iterations, because after reviewing and refining the program preliminaries, the participants had to reconsider the already refined subject themes and identify the course units under each subject theme. Therefore, it was agreed that the activities of reviewing and refining the program preliminaries should come first in the process design. During some iteration sessions, some participants could not differentiate between the core competencies and subject themes. The two terminologies thus required further discussions if they would be merged or not. It was later agreed that they should be separated to ease understanding of the terms.

\section{Results}

In evaluating the ColCuRP, the design criteria in section 5.2 were used. The metrics used for the different aspects included; an acceptable unified view of the curricula, structured activity paths, acceptable outcomes, and facilitated group support. In addition, we also evaluated the efficiency and acceptability of the ColCuRP. Here we assessed whether the ColCuRP provided an optimal way to use the available time for the actual curriculum review meeting to review a curriculum. In other words, whether it reduced the time spent in an actual curriculum review meeting.

\subsection{Presentation and Discussion of Results}

The process outcomes for the ColCuRP are discussed in respect to the evaluation goals. The evaluation of the collaboration process aimed at addressing the needs for the collaborative curriculum review processes. The following results were obtained from the analysis of the participants' responses gotten from the questionnaires and interviews held immediately after the meetings. There were 29 respondents in total, of which 24 were from the questionnaires and 5 from the interviews. The results show that most of the respondents $(88.2 \%)$ had previously participated in the curriculum review. In Table 2, we present the performance results (derived from the interviews and questionnaires) of our ColCuRP evaluation.

Table 2. Performance of CoICuRP

\begin{tabular}{|l|r|r|}
\hline & Mean & $\begin{array}{c}\text { Std. } \\
\text { Deviation }\end{array}$ \\
\hline $\begin{array}{l}\text { Reduces time taken to review the } \\
\text { curriculum - efficiency }\end{array}$ & 2.18 & 1.334 \\
\hline $\begin{array}{l}\text { Reduces the complexity of the } \\
\text { curriculum review process }\end{array}$ & 2.06 & 1.029 \\
\hline $\begin{array}{l}\text { Defines the structure of the } \\
\text { curriculum review process } \\
\text { (identifying tasks systematically) }\end{array}$ & 1.47 & 0.514 \\
\hline $\begin{array}{l}\text { Helps the stakeholders to reach } \\
\text { consensus faster }\end{array}$ & 1.71 & 0.686 \\
\hline $\begin{array}{l}\text { Helps the stakeholders to generate } \\
\text { ideas quickly }\end{array}$ & 1.53 & 0.624 \\
\hline
\end{tabular}

On an acceptable unified view of the curricula, through both observation and questionnaires, we assessed the ColCuRP's ability to support generation of ideas, reduce complexity and help the participants to focus on the matters under deliberation. The responses show that the participants strongly agreed (mean $=1.53$, $\mathrm{SD}=0.624$ ) that the ColCuRP helped them to generate many ideas. The participants also agreed that the ColCuRP was able to bring members with divergent ideas in one meeting to reach consensus on aspects of the curriculum. The results show that the participants agreed $($ mean=2.06, $\mathrm{SD}=1.029)$ that the process allowed them to reach consensus faster.

On efficiency, we assessed whether the ColCuRP would reduce the time spent in an actual curriculum review meeting. In all the four sessions, the participants agreed that if thorough preparations on the pre-process activities are well done, the collaboration process has the potential to greatly improve the time taken to review the curriculum, hence reducing the cost incurred as well; as compared to the previous curriculum review process. These results show that the process was agreeably efficient (mean=2.18, $\mathrm{SD}=1.334$ ). Most of the participants agreed that the collaboration process reduces the time needed to review the curriculum $(83.33 \%)$, though a few $(16.67 \%)$ showed apprehension. Observations revealed that time was lost 
on learning how to use the MeetingWizard GSS (which was not part of the meeting activity) and also on the convergence after the diverging activity. When asked how long it takes to review the curriculum using the traditional process, majority of the participants indicated that it took pretty long, as shown in Table 3. The results show that the ColCuRP can save time in reviewing a curriculum as compared to the existing curriculum review process.

\section{Table 3. Duration of Current Curriculum Review Process}

\begin{tabular}{|l|l|l|l|l|}
\hline \multicolumn{5}{|c|}{ How long does the curriculum review process take? } \\
\hline & Freq & Percent & Valid \% & Cumulative \% \\
\hline $10-15$ days & 4 & 23.5 & 23.5 & 23.5 \\
\hline 1 month & 2 & 11.8 & 11.8 & 35.3 \\
\hline 2 months & 3 & 17.6 & 17.6 & 52.9 \\
\hline 5 months & 8 & 47.1 & 47.1 & 100.0 \\
\hline Total & 17 & 100.0 & 100.0 & \\
\hline
\end{tabular}

On structured activity paths, responses from the participants showed that the step-by-step structure of the activities for a review meeting is a welcome contribution. The participants showed satisfaction with the breakdown of the activities and the support provided by the group support system to anonymously deliberate on a number of issues in a short period of time. In fact, the respondents strongly agreed (mean=1.47, $\mathrm{SD}=0.514$ ) that the ColCuRP provided a systematic task identification means to review meeting activities. One respondent from Makerere University said, "the process looks better than what the college uses. If well managed, it gives a structured way of going through the curriculum review process". This shows the confidence the participant gained from the structured process.

On acceptable outcomes, the ColCuRP should help the stakeholders to achieve their goals and add value to their organization. That is, to support stakeholders to formulate a structure for the reviewed curriculum. In all the four cases, the ColCuRP helped the participants to quickly agree on the matters under deliberation. The responses showed that the participants strongly agreed (mean=1.71, $\mathrm{SD}=0.686$ ) on the ColCuRP's ability to help them to reach consensus faster. The participants confirmed that the ColCuRP helped them to attain the structure of the reviewed curriculum.

On facilitated group support, the ColCuRP aided the facilitator and participants on information sharing and group management. Observations on how the GSS tool (MeetingWizard) provided support; these reveal that the participants didn't know how to use it at the beginning. But on learning, the participants were able to contribute many ideas and share information freely. Using the case of Makerere University, the participants were able to generate, query and discuss a wide range of knowledge areas in a space of only 15 minutes. A record set high of 39 submissions from three workstations using the GSS as compared to the other three previous evaluation cases with an average of 13 submissions from four workstations.

On acceptability, we assessed whether the participants would recommend the adoption of ColCuRP for the future curriculum reviews. The result is shown in Table 4.

Table 4. Recommendation for future use

\begin{tabular}{|l|c|c|}
\hline $\begin{array}{l}\text { Participants who had previously } \\
\text { participated in the curriculum review }\end{array}$ & YES & NO \\
\hline $\begin{array}{l}\text { Do you recommend use of this } \\
\text { collaborative curriculum review } \\
\text { process for future review meetings? }\end{array}$ & $83.33 \%$ & $16.67 \%$ \\
\hline
\end{tabular}

When asked whether they would use the process in the future curriculum reviews, $83.3 \%$ respondents agreed that they would. The undecided respondents $(16.7 \%)$ indicated the need for ample time to review the preliminary documents/reference materials prior to the meeting session. They also showed the need for acquaintance with the use of the GSS tool prior to the review meeting. On what they would improve about the process for future usage, the participants agreed on strictly adhering to the activity time allocations, agreeing and following a clear and concise meeting agenda. They also proposed that the choice of a GSS tool should provide a mechanism for comparing the course units under the new/emerging themes with the course units in the reviewed curriculum and a mechanism to be developed for determining the course unit allocations for the post-review development of the course descriptions.

Although all the four ColCuRP evaluation cases show overall positive results on an acceptable unified view of the curricula, facilitated group support, structured activity paths, acceptable outcomes, efficiency and process acceptability; different participants and academic programs were used. In all evaluation cases, participants performed the same tasks, but for different academic programs, therefore, any variability in the results between evaluation cases cannot negate the overall results of the study. The variations may only be due to factors like facilitator experience and confidence in sharing meetings, previous curricula review experience, pre-meeting preparations, computer literacy levels and familiarity in using other GSS tools.

\section{Conclusion and Future Work}

In this paper, we developed and presented the repeatable collaboration process for the curriculum 
review process. The ColCuRP process design was refined in four iterations using feedback from observations, data logs, questionnaires and interviews. The ColCuRP supports the curriculum reviewers in HEIs to successfully facilitate their review meetings. The results based on the four cases used for the evaluation suggest that the ColCuRP is a feasible solution to the existing challenges in the curriculum review environments. The feedback received from the participants in terms of efficiency, an acceptable unified view of the curricula, structured activity paths, acceptable outcomes, facilitated group support, and acceptability for use, suggest that the ColCuRP has the potential to support the HEIs in the curriculum review process of any academic program. All the respondents agreed that the process can be used repetitively in different HEIs and by different sets of stakeholders without the need for a skilled facilitator(s).

Notwithstanding the promises exhibited by the ColCuRP process, there were some limitations observed. These include among others; the GSS tool limiting the number of participant connections to 5 as well as short of functionalities, a single evaluation cycle of the ColCuRP, hence limiting the performance comparison between the review of similar programs in different HEIs, low number of review participants per session, and minimal curriculum review experience in some of the HEIs. Based on these limitations, our future work will focus on considering more cycles for the evaluation. Also, the review process may be extended to lower institutions of learning such as the secondary and primary schools.

\section{Acknowledgement}

This study was funded in part by the Swedish International Development Cooperation Agency (Sida) and Makerere University

\section{References}

[1] Amiyo, M., Nabukenya, J., and Sol, H.G. (2012) A Repeatable Collaboration Process for Exploring Business Process Improvement Alternatives. Annual Hawaii International Conference on Systems Sciences, IEEE

[2] Baskerville, R. L., (1999). Investigating information systems with action research. Communications of the AIS, 2(3es), 4

[3] Baskerville, R.L., \& Wood-Harper, A.T. (1996). A critical perspective on action research as a method for information systems research. Journal of Information Technology, 11(3), 235-246

[4] Briggs, R.O., De Vreede, G.J., and Nunamaker Jr, J.F. (2003). Collaboration engineering with thinkLets to pursue sustained success with group support systems. Journal of Management Information Systems, 19(4), 31-64

[5] Campbell, D.J. (1988). Task complexity: A review and analysis, Academy of Management Review,13(1), 40-52
[6] Connecticut State Library Digital Collections, (2006 ). A guide to curriculum development-purposes, practices, procedures. Connecticut State Dept. of Education, Division of Teaching and Learning, Bureau of Curriculum and Instruction

[7] Davenport, N.C., Spath, M.L., and Blauvelt, M.J. (2009). A step-by-step approach to curriculum review, Nurse educator, 34(4), 181-185

[8] Eden, C., Jones, S., \& Sims, D., (1983). Messing about in problems: an informal structured approach to their identification and management. Pergamon Press, Oxford, Great Britain

[9] Hevner, A. R., (2007). A three cycle view of design science research. Scandinavian journal of information systems, 19(2), 4

[10] Hevner, A.R., March, S.T., Park, J., \& Ram, S. (2004). Design science in information systems research. MIS Quarterly, 28(1), 75-105

[11] Huyghe, S., Totté, N., \& Verhagen, A. (2013). Building the curriculum in Higher Education: a conceptual framework. Proceedings of the Enhancement and Innovation in Higher Education Conference, 11-13

[12] Kamal, M., Davis, A.J., Nabukenya, J., Schoonover, T.V., Pietron, L.R. and De Vreede, G.J. (2007). Collaboration engineering for incident response planning: process development and validation. 40th Annual Hawaii International Conference on Systems Sciences, IEEE

[13] Kolfschoten, G.L., Briggs, R.O., De Vreede, G.J., Jacobs, P.H., and Appelman, J.H. (2006). A conceptual foundation of the thinkLet concept for Collaboration Engineering. International Journal of Human-Computer Studies, 64(7), 611-621

[14] McNay, M. (2009). Western Guide to Curriculum Review. The University of Western Ontario Teaching Support Centre

[15] Nabukenya, J. (2012). Combining case study, design science and action research methods for effective collaboration engineering research efforts. Annual Hawaii International Conference on Systems Sciences, IEEE

[16] Nabukenya, J., van Bommel, P., and Proper, H. A. (2008). Repeatable collaboration processes for mature organizational policy making. Proceedings of the 14th Collaboration Researchers' International Workshop on Groupware (CRWIG08), 2008, 217-232

[17] Nakakawa, A., (2012). A Collaboration Process for Enterprise Architecture Creation, PdD Thesis, Radboud University Nijmegen, The Netherlands

[18] Nunamaker, J.F., Briggs, R.O., Mittleman, D.D., Vogel, D.R., and Pierre, B. A. (1996). Lessons from a dozen years of group support systems research: A discussion of lab and field findings. Journal of management information systems, 13(3), 163-207

[19] Pare, G. (2004). Investigating information systems with positivist case research. The Communications of the Association for Information Systems, 13(1), 57

[20] Vreede, G.J.de., \& Briggs, R.O. (2005). Collaboration engineering: designing repeatable processes for high-value collaborative tasks. Proceedings of the 38th Annual Hawaii International Conference on System Sciences, IEEE, 17c-17c

[21] Vreede, G.J.de. (2014). Two Case Studies of Achieving Repeatable Team Performance through Collaboration Engineering. MIS Quarterly Executive, 13(2)

[22] Yin, R. K. (2013). Case study research: design and methods, $5^{\text {th }}$ Ed. Thousand Oaks, CA: Sage Publications 